\title{
DETACHMENT WAVES AND THE ONSET OF FRICTIONAL SLIP
}

\author{
Shmuel M. Rubinstein, Gil Cohen, and Jay Fineberg \\ The Racah Institute of Physics, The Hebrew University of Jerusalem, Givat Ram, Jerusalem Israel
}

\begin{abstract}
We perform real-time measurements of the net contact area between two blocks of like material at the onset of frictional slip. We show that the process of interface detachment, which immediately precedes the inception of frictional sliding, is governed by three different types of detachment fronts. These crack-like detachment fronts differ by both their propagation velocities and by the amount of net contact surface reduction caused by their passage. The most rapid fronts propagate at intersonic velocities but generate a negligible reduction in contact area across the interface. Sub-Rayleigh fronts are cracklike modes which propagate at velocities up to the Rayleigh wave speed, $V_{R}$, and give rise to an approximate $10 \%$ reduction in net contact area. The most efficient contact area reduction $(\sim 20 \%)$ is precipitated by the passage of "slow detachment fronts". These fronts, which have not previously been observed, propagate at velocities over an order of magnitude lower than $V_{R}$. They are generated, in conjunction with intersonic fronts, by the sudden arrest of sub-Rayleigh fronts. No overall sliding occurs until either of the slower two fronts traverses the entire interface, and motion of the leading edge of the interface is initiated. Slip at the trailing edge of the interface accompanies the motion of both the slow and sub-Rayleigh fronts. We might expect these modes to be important in both fault nucleation and earthquake dynamics.
\end{abstract}

\section{INTRODUCTION}

The dynamics of frictional slip have been studied for hundreds of years, yet many aspects of these everyday processes are not understood. One such process is the onset of slip. First described by Coulomb and Amontons as the transition from static to dynamic friction, the onset of frictional slip is central to fields as diverse as physics, tribology, the study of earthquakes and fracture. The dynamics of friction have, traditionally, been considered to be governed by processes that occur at slow time scales, when the entire slider is in motion. Here we study the detailed processes that occur at the interface between two blocks of like material, immediately prior to the onset of frictional sliding. These processes, in which extremely short time scales are dominant, take place via 3 different types of detachment fronts which propagate along the interface. These rapid processes are fundamental to fault nucleation and the onset of slip in earthquake dynamics.

Our experiments describe the dynamics of two brittle acrylic (PMMA) blocks separated by a rough interface (each surface was roughened to an approximate 
roughness of $1 \mu \mathrm{m} \mathrm{rms}$ ), consisting of many discrete randomly distributed microcontacts. Slip is initiated by slowly increasing applied shear force, $F_{S}$, while loading the blocks with a constant normal force, $\mathrm{F}_{\mathrm{N}}$. The dynamics of the interface, described below, are measured by means of real-time visualization of the net contact area formed by the rough interface. The experimental apparatus, schematically shown in Figure 1a, measures the net contact area between the two rough surfaces that form the interface between the two blocks. The interface is illuminated by a laser sheet whose angle of incidence is much larger than the critical angle needed for total internal reflection from the acrylic-air interface. For the experimental conditions used, light will only effectively traverse the interface at points of contact. Thus, the intensity, I, at each point along the interface is proportional to the net contact area at that spatial location. The intensity as a function of space is then imaged by a fast camera, which enables us to visualize the instantaneous net contact area along the entire interface at rates of up to $4 \mu \mathrm{sec}$ per frame. As Figure $1 \mathrm{~b}$ shows, the spatially integrated transmitted intensity, hence the total net contact area along the interface, is a linear function of the applied normal stress, providing a direct validation of the Bowden and Tabor picture of friction (Bowden [1], Persson [2]).

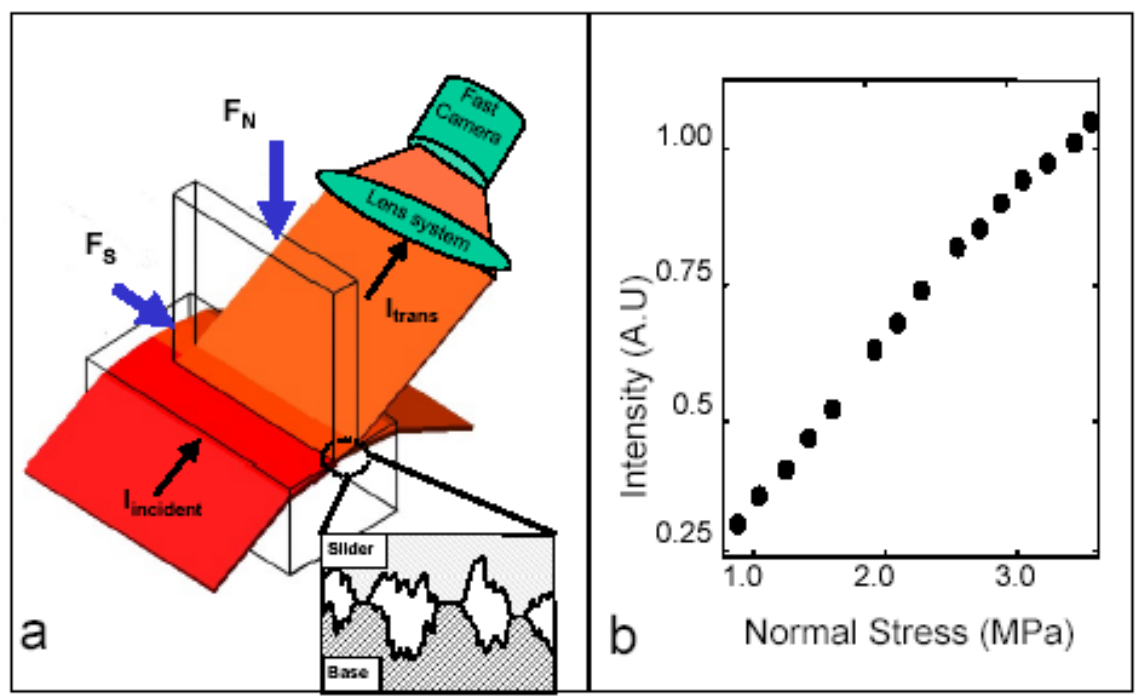

Figure 1: (a) A schematic view of the experimental apparatus, in which a laser sheet is incident on a rough interface (inset) at an angle beyond the angle for total internal reflection from a PMMA-air interface. Light can only traverse the interface at the actual points of contact. The transmitted light, imaged on a fast camera, is therefore proportional to the net contact area at each point. (b) The integrated intensity of light, hence the total net contact area, is proportional to the applied normal stress, $\mathrm{F}_{\mathrm{N}}$ as predicted in the Bowden and Tabor theory of friction. 


\section{RESULTS}
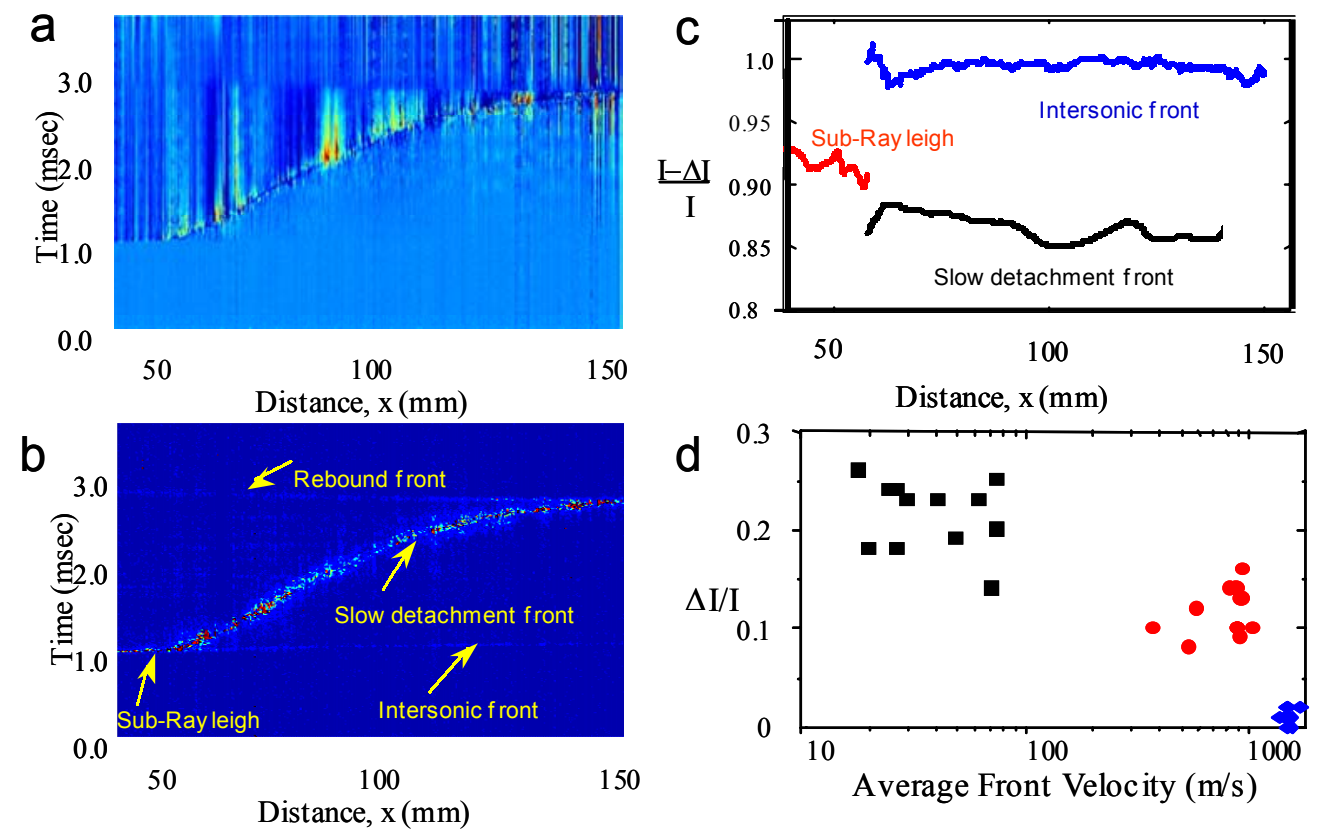

Figure 2: The dynamics of slip, prior to overall sliding, take place via the interplay between 4 different types of coherent crack-like fronts. (a) The transmitted intensity $\mathrm{I}(\mathrm{x}, \mathrm{t})$, averaged in the y direction, of a typical experiment as a function of time. Successive lines spanning the $150 \mathrm{~mm}$ long interface were acquired at $10 \mu \mathrm{sec}$ intervals. These intensity measurements, normalized by their initial values at each spatial point, are color-coded to reflect the change in the contact area at each spatial point as a function of time. Hot (cold) colors reflect increased (decreased) net contact area. (b) The temporal derivative, dI/dt, of the data presented in (a), color coded as above. The 4 different types are labeled within the plot. The visibility of the fronts, which can also be seen in (a), is enhanced by the derivation. (c) The relative drop in contact area, as indicated by the intensity drop (I- $\Delta \mathrm{I}) / \mathrm{I}$ across each of the three forward-propagating fronts shown in (a). (d) The contact area reduction, $\Delta \mathrm{I} / \mathrm{I}$, in a number of different experiments for slow detachment fronts (squares), sub-Rayleigh fronts (circles) and intersonic fronts (diamonds) as a function of their measured propagation velocities. Note both the large differences in velocities and significant differences in the contact area reductions of the different fronts. 
The onset of slip is immediately preceded by the interplay of three different types of coherent crack-like detachment fronts, as shown in Figure 2a and Figure 2b. Detachment is initiated by the first of these detachment fronts, the "sub-Rayeigh" fronts, which propagate at rapid subsonic velocities up to and including the Rayleigh wave speed, $V_{R}$. Sub-Rayleigh fronts lead to an approximate $10 \%$ decrease in the local contact area, immediately upon their passage. These fronts can be identified with earthquakes that propagate in the $0.2-$ $0.8 \mathrm{~V}_{\mathrm{R}}$ range. Upon detachment initiation, these fronts rapidly accelerate until arriving at velocities in the vicinity of $V_{R}\left(V_{R}=935 \mathrm{~m} / \mathrm{s}\right.$ in PMMA). At approximately $\mathrm{V}_{\mathrm{R}}$, these sub-Rayleigh fronts arrest and are replaced by two additional types of fronts, which are simultaneously emitted.

The more rapid of these fronts are intersonic fronts, which propagate at speeds which are considerably higher than the shear wave velocity. These types of fronts have been observed in recent experiments (Rosakis [5], Xia [6]) and calculations (Gao [3], Needleman, 4]) of explosively induced shear fracture. There is also evidence for such fronts in the seismic records (Bouchon [7]) of recent earthquakes (e.g. Izmit, 1999). We will show that, although these waves traverse the entire length of the interface, they give rise to minimal (1-2\%) detachment of the net contact area and negligible slip.

The second type of front which is emitted upon arrest of the sub-Rayleigh fronts is a new type of front, a "slow detachment front", which propagates an order of magnitude more slowly than the sub-Rayleigh fronts. Our measurements suggest that slow detachment fronts nearly always occur; either as isolated processes or in conjunction with the more rapid modes. These fronts are the dominant mechanism for interface detachment, leading to about a $20 \%$ reduction in contact area upon their passage. The slow fronts also cause a significant amount of slip while in motion, but their acoustic signatures are significantly weaker than those of the faster modes. Recent observations of slow or "silent" earthquakes (Crescentini[8], Linde [9], Rogers [10]), in which significant slip was observed with a minimal acoustic signature, may therefore be analogous to these fronts.

No overall sliding of the two blocks occurs until either of the slower two modes traverses the entire interface. The onset of sliding, which is initiated by the detachment fronts described above, is the transition from static to dynamic friction. The 10-20\% reduction in the static verses dynamic coefficients of friction for PMMA is consistent with the overall $12 \%$ average reduction in the net contact area that we observe prior to the onset of sliding. Thus, this work elucidates both the mechanisms responsible for the transition from static to dynamic friction as well as possible key processes that take place in dynamics of fault nucleation. 


\section{REFERENCES}

1. Bouchon, M., M.P. Bouin, H. Karabulut, M.N. Toksoz, M. Dietrich, and A.J. Rosakis, How fast is rupture during an earthquake? New insights from the 1999 Turkey earthquakes, Geophys. Res. Lett., 28 (14), 2723-2726, 2001.

2. Bowden, F.P., and D. Tabor, The Friction and Lubrication of Solids, Oxford Univ. Press, New York, 2001.

3. Gao, H.J., Y.G. Huang, and F.F. Abraham, Continuum and atomistic studies of intersonic crack propagation, J. Mech. and Phys. of Sol., 49 (9), 21132132, 2001.

4. Needleman, A., An analysis of intersonic crack growth under shear loading, J. Appl. Mech., 66 (4), 847-857, 1999.

5. Persson, B.N.J., Sliding Friction Physical Principles and Applications, Springer-Verlag, New York, 2000.

6. Rosakis, A.J., O. Samudrala, and D. Coker, Cracks faster than the shear wave speed, Science, 284 (5418), 1337-1340, 1999.

7. Xia, K., A.J. Rosakis, and H. Kanamori, Laboratory Earthquakes: The SubRaleigh-to-Supershear Rupture Transition, Science, 303 (19), 1859-1861, 2004.

8. Crescentini, L., A. Amoruso, and R. Scarpa, Constraints on slow earthquake dynamics from a swarm in central Italy, Science, 286, 2132-2134, (1999).

9. Linde, A.T., and I.S. Sacks, Slow earthquakes and great earthquakes along the Nankai trough, Earth and Planetary Science Letters, 203 (1), 265-275, 2002.

10. Rogers, G., and H. Dragert, Episodic tremor and slip on the Cascadia subduction zone: The chatter of silent slip, Science, 300 (5627), 1942-1943, 2003. 